\title{
A não efetividade do hedge para o boi gordo
}

\author{
Waleska de Fátima Monteiro ${ }^{1}$ \\ Marcos Aurelio Rodrigues ${ }^{2}$ \\ Alexandre Florindo Alves ${ }^{3}$ \\ José Luiz Parré4
}

\begin{abstract}
Resumo: O objetivo desse artigo é estimar a efetividade e razão ótima do hedge do boi gordo brasileiro, nas praças de Araçatuba, Campo Grande, Três Lagoas, Cuiabá, Goiânia e Noroeste do Paraná, no período de 2002 a 2008. O procedimento metodológico considera as relações de cointegração entre os mercados à vista e futuro, com abordagens dinâmicas para as variâncias e covariâncias. Os resultados não apresentam condições para um hedge efetivo, ao considerar os retornos das séries em logaritmo e não os preços em nível, uma vez que a estacionariedade é atingida na primeira diferença; a adição de um termo de correção de erro, para garantir a relação de longo prazo; assim como correlações e variâncias variantes no tempo. Portanto, o contrato futuro de boi gordo não cumpre seu propósito de diminuição de risco aos hedgers.
\end{abstract}

Palavras-chaves: Efetividade; Razão ótima de hedge; Boi Gordo.

Classificação JEL: $C_{13}, G 13$

\section{The non-effectiveness of hedge for live cattle}

\footnotetext{
Abstract: In this paper we aim to estimate the effectiveness and optimal hedge ratio of the Brazilian live cattle in the markets of Araçatuba, Campo Grande, Três Lagoas, Cuiabá, Goiânia and Northwest Paraná, between 2002 and 2008. The methodological procedure considers the cointegration relations between spot and future markets, with a dynamic approach for the variance and covariance. The

1 Doutoranda em Economia na Universidade de Brasília (UNB). Email: wfmonteiroo5@gmail.com 2 Doutorando em Economia Aplicada na Escola Superior de Agricultura "Luiz de Queiroz" (ESALQ) - Universidade de São Paulo (USP). Email: rodriguesmarcosaurelio@gmail.com

3 Doutor em Economia Aplicada na Escola Superior de Agricultura "Luiz de Queiroz" (ESALQ) - Universidade de São Paulo (USP), Professor Associado, departamento de Economia na Universidade Estadual de Maringá (UEM). Email: afalves@uem.br

4 Doutor em Economia Aplicada na Escola Superior de Agricultura "Luiz de Queiroz" (ESALQ) - Universidade de São Paulo (USP), Professor Associado, departamento de Economia na Universidade Estadual de Maringá (UEM). jlparre@uem.br
} 
MONTEIRO, W. F.; RODRIGUES, M. A;.ALVES, A. F.; PARRÉ, J. L. A não efetividade do hedge...

results do not support an effective hedge even if we use the logarithm of the returns (instead of the price level) bacause of the first-difference stationarity; add an error correction term to guarantee the long-term relationship; and consider time-varying correlations and variances. Therefore, the Brazilian live cattle futures contract does not fulfill its purpose of risk reduction for hedgers.

Keywords: Effectiveness; Optimal hedge ratio, Live Cattle.

Classificação JEL: $C 13, G 13$

\section{Introdução}

Os mercados futuros de commodities foram desenvolvidos para atender produtores e comerciantes expostos a riscos de preços, nos períodos de escassez e superprodução do produto negociado, reduzindo o risco de flutuação dos preços da mercadoria. Por conseguinte, ajudam na comercialização das commodities agropecuárias, minerais e financeiras. Dentre suas funções, a principal é proteger os interessados de variações desfavoráveis, através da fixação do preço de compra ou de venda em determinada data futura, com a intenção de minimizar os riscos de perda. Como corolário, constitui-se uma ferramenta crucial para o gerenciamento efetivo do risco nos mercados imperfeitos em que os agentes operam. Pode proporcionar proteção patrimonial, melhor remuneração do seu produto e maior estabilidade na atividade e competitividade.

Entretanto, Hull (2006) ressaltou que os derivativos são instrumentos muito versáteis, e é por esta versatilidade que causam contratempos. Às vezes, as empresas que pretendem "hedgear" seu risco tornam-se especuladores, consciente ou inconscientemente, extrapolando a necessidade de "hedgear". Os resultados podem ser desastrosos. Para evitar os problemas advindos da especulação intencional ou não intencional, é importante, tanto para agentes financeiros como não financeiros, monitorar diariamente seus derivativos para que sigam o único propósito de hedge, impondo limites de riscos. Situações como essas são relativamente novas no Brasil, mas comuns no exterior. Portanto, demandam calcular o número de contratos futuros que devem ser negociados, a razão de hedge, uma vez que sua efetividade, redução de risco máximo, pode ser obtida somente com a razão de hedge corretamente estimada. Os resultados podem ser influenciados por correlações entre os retornos futuros e à vista, os métodos para estimá-lo, bem como o risco de base.

Para a atividade pecuária, o risco de preços fica evidente no momento em que o produtor engorda seus animais sem saber qual o preço que receberá no futuro, pois existirá o custo de engorda, no entanto, o valor a ser recebido pode não cobri-lo. No decorrer do período de engorda os preços podem variar significativamente, sob impacto dos diversos fatores que influenciam a oferta e demanda do produto, como: variações nos preços da carne suína ou do 
frango; aumento dos preços dos insumos utilizados no processo de engorda; interrupção das compras externas por receio de doenças, etc (BM\&F, 2007). Estes podem ser suficientes para que o preço a receber não remunere corretamente o pecuarista ou cubra os custos de sua produção.

Diante deste cenário, faz-se necessário investigar a efetividade do hedge no mercado de boi gordo, uma vez que o Brasil é o maior exportador mundial de carne bovina, além de ser o produto agropecuário com maior liquidez na BM\&FBOVESPA, conforme seus boletins. O objetivo do presente estudo é estimar a efetividade e razão ótima do hedge, ao considerar as relações de cointegração de longo prazo, entre os mercados à vista e futuro, com abordagens dinâmicas para as variâncias e covariâncias dos preços do boi gordo brasileiro. Difere da literatura nacional nos seguintes aspectos i) assume uma distribuição dos erros bivariada t de Student, ii) considera um termo de correção de erro dentro da estimativa multivariada GARCH BEKK com alvo na variância iii) as razões e efetividades do hedge são analisadas dentro e fora da amostra.

\section{Aspectos sobre o mercado futuro}

Sejam $s^{1}$ e $s^{c}$ o preço à vista nos períodos $t_{1}$ e $t_{\varsigma^{\circ}}$ O ganho ou a perda de uma posição não "hedgeada", ${ }_{U}$, é $\left(s^{2}-s^{1}\right)$ e, para uma posição "hedgeada", $H$, é $\left(s^{2}-s^{1}\right)-\left(f^{1}-f^{2}\right)$, em que $f$ é a notação do preço futuro. Apesar do comovimento entre os preços à vista e futuros, o valor absoluto de $H$ é menor que $U$, deste modo, var $(H)<\operatorname{var}(U)$, questão explicada pela variação da base.

A base é a diferença entre os preços à vista e futuros. A volatilidade da base é menor que a dos preços futuros e sua correlação quase sempre negativa. Logo, a variância mínima da razão de hedge será, em geral, abaixo de 1 (Alexander, 2008). Entretanto, como existe a convergência do preço futuro com o à vista, no dia de encerramento será igual a 1. Portanto, no tempo $t$ a base é definida como $B(t, T)=s(t)-f(t, T)$. tal que $B$ é a base, $t$ o tempo, $s$ o preço à vista, $f$ o preço futuro e $T$ o vencimento. Quando os preços à vista aumentam mais que os futuros, a base aumenta. Este movimento é definido como fortalecimento da base. Porém, quando os preços futuros aumentam mais que os à vista, a base reduz. Isto define o enfraquecimento da base.

Em geral, observa-se que os preços à vista e futuro se movem na mesma direção. Entretanto, além de não ser simultâneo, não ocorre na mesma intensidade (Silveira; Ferreira Filho, 2003). Estes movimentos podem ser imprevisíveis no que diz respeito à base, por isso, define-se risco de base. Diante deste fato, é perceptível que o mercado futuro não esgota totalmente o risco quanto à oscilação de preços, mas reduz de forma segura. 
MONTEIRO, W. F.; RODRIGUES, M. A;.ALVES, A. F.; PARRÉ, J. L. A não efetividade do hedge...

No intuito de balancear de forma adequada as posições nos mercados físicos e futuros, é imprescindível saber a relação ótima entre eles, pois os movimentos nos preços não são independentes. Se fossem, seria possível negligenciar a volatilidade do portfólio. O modelo teórico sobre hedge desenvolvido por Ederington (1979) visou solucionar esta equação, ao relacionar a variação do preço à vista com o preço futuro, por meio de Mínimos Quadrados Ordinários (MQO). Propôs a variância mínima da razão de hedge (VMRH), razão entre a covariância do preço futuro e à vista com a variância do preço futuro. $\mathrm{O}$ propósito de um agente econômico ao fazer hedge é minimizar a variância de seu portfólio, logo, a VMRH será a razão de hedge ótima (RHO), dada por

$$
h_{t}^{*}=\frac{\sigma_{s f, t}}{\sigma_{f, t}^{2}},
$$

Contudo, se os preços mudam no tempo, suas variâncias e covariâncias não são constantes. A solução proposta por Ederington (1979) desconsidera as variâncias e covariâncias condicionais, dada as informações disponíveis no período $t$. Portanto, é necessário um estimador forwardlooking para a razão de hedge. Kroner e Sultan (1993) passaram a estimá-la como $h_{t}^{*}=\frac{\sigma_{s f, t+1}}{\sigma_{f, t+1}^{2}}$.

Similar à anterior, exceto que os momentos incondicionais às variações no tempo são repostas por momentos condicionais e variantes no tempo. Portanto, a razão de hedge passa a mudar à medida que novas informações chegam aos agentes.

Ederington (1979) introduziu uma simples maneira de mensurar a efetividade do hedge, como sendo a redução percentual da variância ao fazer o hedge, relativo a não fazê-lo. Esta redução pode ser assim calculada como

$$
E=\frac{\operatorname{var}(U)-\operatorname{var}(H)}{\operatorname{var}(U)},
$$

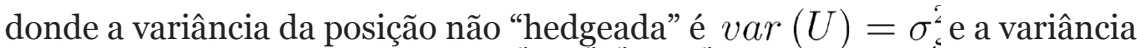
da posição "hedgeada" var $(H)=\sigma_{s}^{2}+h^{2} \sigma_{f}^{2}-2 h^{2} \sigma_{s f}$.

\section{Especificações das razões e efetividades de hedge}

Uma das questões teóricas que envolvem hedge é a determinação de sua razão ótima, a qual depende de uma função a ser otimizada. Assim como Johnson (1960), Ederington (1979), Myers e Thompson (1989) e Kroner e Sultan (1993), este estudo visa a minimização da variância de um portfólio "hedgeado". A revisão de literatura centra nos aspectos sobre minimização dos riscos inerentes a carteiras "hedgeadas", fator preponderante na análise do hedger. A efetividade do hedge foi motivo de vários estudos nos mercados 
acionários, cambiais, agrícolas e pecuários. Os autores comparavam qual modelo ajusta melhor à volatilidade encontrada nesses mercados. A fim de minimizar os riscos da carteira, recorriam a modelos estáticos, que desconsideram a dinâmica das variâncias e covariâncias dos preços e assumem uma razão de hedge constante no tempo.

As aplicações empíricas iniciam com o modelo de hedge perfeito, cujo preço futuro reflete com exatidão o preço à vista, isto é, o coeficiente da correlação entre preço futuro e à vista seja igual a 1 e seus desvios padrão os mesmos. Esta suposição é muito forte, pois a razão de hedge será igual a 1. Nas palavras de Alexander (2008) o hedger acredita que os preços de ambos os mercados possuem uma correlação igual à volatilidade do mercado futuro com respeito ao à vista. Uma forte restrição à crença do hedger. No mundo real, esta relação é praticamente impossível. Entretanto, muitos agentes realizam o hedge de suas exposições utilizando uma razão de hedge igual a 1, chamada pela literatura de hedge ingênuo. Ao relaxar esta suposição, dando mais realidade à volatilidade do mercado, empregam o modelo de Mínimos Quadrados Ordinários (MQO). Amplamente utilizado na literatura, estima de forma simples a razão de hedge, com a mudança do preço à vista sobre a mudança do preço futuro.

Apesar do método tradicional ser indubitavelmente convidativo, devido à sua fácil aplicação, sofre de inúmeras limitações. A estimação da razão de hedge pelo modelo MQO, proposta por Ederington (1979) seria apropriada para fornecer essa razão, entretanto, assume que é constante ao longo do tempo, além de não considerar a correlação serial. A questão relevante deste tipo de estimação da razão de hedge é a falta de especificação do modelo, que não considera o conteúdo informacional. O estudo de Lien e Tse (2002) ressaltou que o principal problema deste modelo situa-se na dependência não condicional dos segundos momentos, enquanto a "verdadeira" razão de hedge é baseada na dependência condicional dos segundos momentos. Segundo Silveira e Ferreira Filho (2003), a estimação por MQO desconsidera o conteúdo informacional, pois apresenta apenas as variâncias e covariâncias incondicionais entre os preços futuros e à vista, e a regra da razão de hedge ótima está, claramente, condicionada às informações disponíveis no momento da realização das estratégias de hedge. Os estudos de Herbst, Kare e Caples (1989), Yang e Allen (2005) e Ye e Chen (2006) demonstram que o modelo MQO é inválido, pelo fato dos resíduos serem autocorrelacionados, uma vez que a maioria das regressões de séries temporais no modelo MQO têm problema de autocorrelação positiva. A fim de eliminar a autocorrelação serial, entre os preços futuros e à vista, os autores utilizaram o vetor autoregressivo (VAR).

O modelo vetorial autoregressivo tem a deficiência de não captar as informações de como se movimentam os preços no longo prazo, relevantes para realizar o hedge, dado que podem influenciar os movimentos do curto prazo. Dessas acepções, Kroner e Sultan (1993) mostraram que a regressão dada pela 
MONTEIRO, W. F.; RODRIGUES, M. A;.ALVES, A. F.; PARRÉ, J. L. A não efetividade do hedge...

equação no estilo VAR é mal especificada quando os preços à vista e futuro são cointegrados, pois ignora a correção de erro, isto é, exclui o impacto do desvio ao equilíbrio do último período. Lien e Zhang (2008) argumentaram a existência de cointegração entre os preços à vista e futuro. Sendo assim, para corrigir os erros de especificação torna-se razoável considerar um vetor de correção de erros. Segundo Müller et al. (2008) os modelos estáticos atuam sobre a hipótese de variância incondicional, isto é, variância homocedástica, argumentada como não apropriada, pois as condições de riscos dos mercados se alteram. Deste modo, faz-se necessário o ajustamento das posições de forma dinâmica.

Para superar esta limitação, Kroner e Sultan (1993), Bitencourt et al. (2006) e Müller et al. (2008) estimaram por modelos multivariados GARCH, que permitem variações na razão de hedge. De acordo com Brooks (2008) os preços das commodities são caracterizadas por matrizes de covariância que variam no tempo. Logo, existe um consenso geral que o uso de modelos MGARCH possuem performances superiores, evidenciados por portfólios com menores volatilidades, comparado com os modelos de hedge que não capturam a variação no tempo na matriz de covariância, tais como MQO, VAR e VEC.

No Brasil, Martins e Aguiar (2004) estudaram a efetividade do hedge na CBOT para diversas regiões brasileiras produtoras de soja. Os contratos do segundo semestre foram mais efetivos para os produtores brasileiros. No caso de quebra na safra norte-americana, os de novembro e janeiro tornam-se efetivos e as regiões com maior redução de risco são as próximas aos portos de exportação.

O estudo de Fileni et al. (1999) versou sobre o risco de base e efetividade do hedge em diversas praças brasileiras produtoras de café. Como resultados, os riscos de base são semelhantes e, através do método tradicional proposto por Ederington (1979), mostrou que os contratos futuros de café na BM\&F são eficientes na redução do risco. Evidência semelhante ao estudo de Nogueira, Aguiar e Lima (2002), que considerou operações de hedge sobre café efetuadas nas bolsas BM\&F e Coffee, Sugar \& Cocoa Exchange (CSCE), para séries diárias, semanais e quinzenais. Verificaram maior efetividade na BM\&F que na CSCE. Outra iniciativa no mercado brasileiro de café foi apresentada por Müller et al. (2008). As estratégias de hedge foram modeladas por MQO, VEC, BEKK, ingênuo, e sem hedge. Os resultados evidenciam como pior estratégia não fazer hedge. A ingênua não foi uma boa estratégia, a BEKK foi a de maior redução do risco e a estratégia por MQO aplicável a agentes com pequena escala de comercialização, pois demanda pouco tempo de gerenciamento e pode ser realizada pelo próprio agente. É importante salientar que este método fornece uma razão constante, pois as variâncias e covariâncias são incondicionais, não considera a correlação serial bem como conteúdo informacional.

A análise de efetividade do hedge para o mercado brasileiro de gado bovino foi estudada por Silveira e Ferreira Filho (2003). Examinaram operações de cross hedge do bezerro na BM\&F, nas principais regiões de comercialização 
brasileira desta commoditie. Concluem a partir do modelo auto regressivo, que os pecuaristas que fazem hedge de bezerro, com contratos futuros de boi gordo, não possuem um instrumento eficiente contra variações de preços. Guimarães (2005) chegou a conclusão semelhante, em estudo sobre hedge do boi gordo, realizado com o indicador ESALQ/BM\&FBOVESPA. Tanto a razão de hedge como a efetividade resultaram em valores relativamente baixos, mesmo quando considerada a cointegração. Segundo o autor, de forma geral, os agentes na cadeia pecuária tendem à especulação e não tomam posições para administrar o risco, seja pelo custo ou pelo grau de aversão ao risco.

Destacam-se na literatura Bitencourt et al. (2006) que analisaram o mercado de boi gordo brasileiro por método dinâmico. Assim como Guimarães (2005), a base de dados foi construída com preços à vista, indicador ESALQ/ BM\&FBOVESPA, e preços futuros do boi gordo na BM\&FBOVESPA. A razão de hedge ótima foi estimada pelo modelo MQO e BEKK, o qual propiciou melhor desempenho na redução da variância. $\mathrm{O}$ artigo de Gonçalves et al. (2008) evidenciou a cointegração entre os preços à vista e futuros do boi gordo na região noroeste do Paraná. A efetividade do hedge encontrada, através do modelo VEC, foi baixa. Mais um indício que o contrato futuro não proporciona uma gestão de risco adequada aos integrantes da cadeia bovina. Sugerem que o risco de base pode ser a explicação para este resultado.

\section{Metodologia}

Para o procedimento empírico, foram consideradas seis praças, dentre os dez estados com maior rebanho bovino efetivo no país ${ }^{1}$ : Araçatuba no estado de São Paulo, Campo Grande e Três Lagoas no Mato Grosso do Sul, Cuiabá no Mato Grosso, Goiânia em Goiás e Noroeste do Paraná no estado do Paraná. A base de dados foi construída com os preços à vista, obtidos no $\mathrm{CEPEA}^{2} \mathrm{e}$ futuros do primeiro vencimento na BM\&FBOVESPA. Segundo Guimarães (2005), considerar estratégias de hedge com o primeiro vencimento do contrato de boi gordo faz sentido aos agentes intermediários da cadeia com alta rotatividade da mercadoria. O período observado inicia-se em 02/01/2002 e termina em 24/06/2008, sendo desconsiderados os feriados nacionais e os que afetaram os pregões. Para cada praça, o número de observações foi $\approx$ 1600, sendo retiradas 100, fora da amostra, para efeitos de predição.

O cômputo da razão de hedge segundo a equação (3) é fundamentada na variância e covariância de variáveis aleatórias em log normal, padrão em finanças com tempo contínuo (Alexander, 2008). A literatura acadêmica financeira geralmente utiliza o log-retorno, desde que seja a razão logarítmica do preço atual com a do período anterior. Possui a propriedade de ser tratada como retornos contínuos - portanto pode ser facilmente comparado e combinado (Brooks, 2008). 
Os retornos à vista $S=\ln (\Delta s)=\ln \left(\frac{s_{t}}{s_{t-1}}\right)$ e futuro $F=\ln (\Delta f)=\ln \left(\frac{f_{t}}{f_{t-1}}\right)$, foram calculados através da divisão entre o preço do dia $s_{t}$ e anterior $s_{t-1}$, para os preços à vista, e entre o ajuste atual $f_{t}$ e o anterior $f_{t-1}$, para os preços futuros. Com os resultados em percentuais obtidos, é computado o logaritmo neperiano ln. Além disso, é admitido que a condição martingal seja satisfeita.

Os valores encontrados são usados nos métodos econométricos, descritos e estimados, com propósito de computar a razão ótima e efetividade do hedge. Ao partir dos pressupostos revistos na sesão anterior, não são consideradas as estimações estáticas, usualmente utilizadas nos artigos empíricos, pois não possuem as propriedades estatísticas desejáveis. Assim, apenas a modelagem multivariada GARCH proposta por Engle e Kroner (1995), que se adequam às características levantadas, são efetuadas. As performances são comparadas entre si, ou seja, a efetividade das estratégias de hedge, por meio da modelagem BEKK diagonal.

Segundo Fama e French (1987) a base reflete a convergência dos preços à vista e futuros. As evidencias bem documentadas de cointegração entre os mercados de boi gordo, sugeridas por Guimarães (2005), Zilli et al. (2008), Gonçalves et al. (2008) e Abitante (2008) dão suporte ao uso da base no período $t-1$ , $\left(s_{t-1}-\delta f_{t-1}\right)$, adicionada como termo de correção de erro na equação dinâmica, tal que $\delta=1$. A proposta de Kroner e Sultan (1993) impõe restrições de longo prazo dentro do modelo de curto prazo, possui poder preditivo sobre os retornos e ao conduzirem testes de cointegração, invariavelmente foi encontrado $\delta \approx 1$. Gaio, Castro e Oliveira (2005) encontraram valores para $\delta \approx 1$. Portanto, o termo de correção de erro utilizado será a base em $t-1$. No intuito de conduzir os procedimentos variantes no tempo, para as razões de hedge, primeiro, é desejável certificar que as matrizes de correlação não sejam constantes no tempo, por meio do teste de Engle e Sheppard (2001), sob hipótese nula de correlação constante. Rejeitadas, pode-se, portanto, seguir com a modelagem BEKK diagonal dos retornos à vista e futuros, descrita de acordo com a equação seguinte:

$$
H_{t}=C_{0}^{*^{\prime}} C_{0}^{*}+\sum_{k=1}^{K} C_{1 k}^{*^{\prime}} x_{t} x_{t}^{\prime} C_{1 k}^{*}+\sum_{k=1}^{K} \sum_{i=1}^{q} A_{i k}^{*^{\prime}} \varepsilon_{t-1} \varepsilon_{t-i}^{\prime} A_{i k}^{*}+\sum_{k=1}^{K} \sum_{i=1}^{p} G_{i k}^{*^{\prime}} H_{t-i} G_{i k}^{*}
$$

em que $C_{0}^{*}, A_{i k}^{*}$, e $G_{i k}^{*}$ são matrizes de parâmetros $n \times n$ com $C_{0}^{*}$ triangular; $C_{1 k}^{*}$ são matrizes de parâmetros $J \times n$; e o limite $K$ do somatório determina a generalidade do processo. Esta possui a vantagem de requerer menos parâmetros na estimação condicional de variâncias e covariâncias. Como os termos do lado direito da equação são expressos 
em forma quadrática, garantem que a matriz de covariância condicional seja definida positiva. Logo, supera a principal fraqueza do modelo vech.

$\mathrm{O}$ modelo BEKK é estimado sujeito à restrição chamada de alvo na variância. De acordo com Engle e Mezrich (1996) o método reparametriza a equação de volatilidade, ao substituir o intercepto pela variância de longo prazo, forçando a matriz de variância e covariância a tomar um valor particular e plausível dentro da matriz de covariância. Assim, reduz o número de parâmetros e, em muitos casos, provê melhor performance.

\section{Análise dos dados}

Os resultados nesta seção compreendem análises preliminares das séries, testes de estacionariedade, cointegração e de correlação condicional constante. Após o levantamento informacional das séries, são estimados os parâmetros da modelagem BEKK diagonal com alvo na variância. Por conseguinte, tornam-se possíveis os cômputos das razões de hedge, que permitem chegar ao resultado final das efetividades, dentro e fora da amostra.

Foi utilizada a estatística descritiva como etapa inicial na compreensão dos dados de alta frequência, sumarizada na Tabela 1. Como os retornos são computados por primeira diferença dos preços em log, perde-se uma observação e, por hipótese, $E\left(F_{t}\right)=E\left(F_{t+1}\right)=0$, logo, a suposição do processo martingal é razoável, pois os retornos são centrados em zero. Apesar disso, o mercado futuro foi menos estável que os à vista. Ao comparar os desvios padrões, estes foram similares nas diversas praças e inferiores ao mercado futuro.

As evidências nos ativos financeiros indicam obliquidade com mesmo sinal entre os mercados à vista e futuro. Entretanto, no boi gordo aparenta ser diferente, pois existe um descompasso entre os mercados. Ocorre assimetria negativa nos retornos à vista e positiva nos futuros. Outra característica divergente é o excesso de curtose dos retornos futuros serem maiores que dos à vista, exceto em Campo Grande e Três Lagoas. As distribuições incondicionais univariadas revelam a não normalidade, pois apresentaram distribuições assimétricas, leptocúrticas e estatística Jarque-Bera significante. Este conjunto de evidências justificam o uso da distribuição $t$ de student nas modelagens, para contemplar as características das séries.

A existência de correlação elevada entre os ativos é condição necessária para o hedge ser efetivo. Apesar da elevada correlação dos preços e próxima a um, não faz sentido estabelecer alguma inferência pois as médias e variâncias podem não ser definidas. Só faz sentido estatístico explicar o comportamento dos retornos. Em todas as séries a correlação dos retornos é baixa e servem como primeiro indício de não efetividade do hedge. 
MONTEIRO, W. F.; RODRIGUES, M. A;.ALVES, A. F.; PARRÉ, J. L. A não efetividade do hedge...

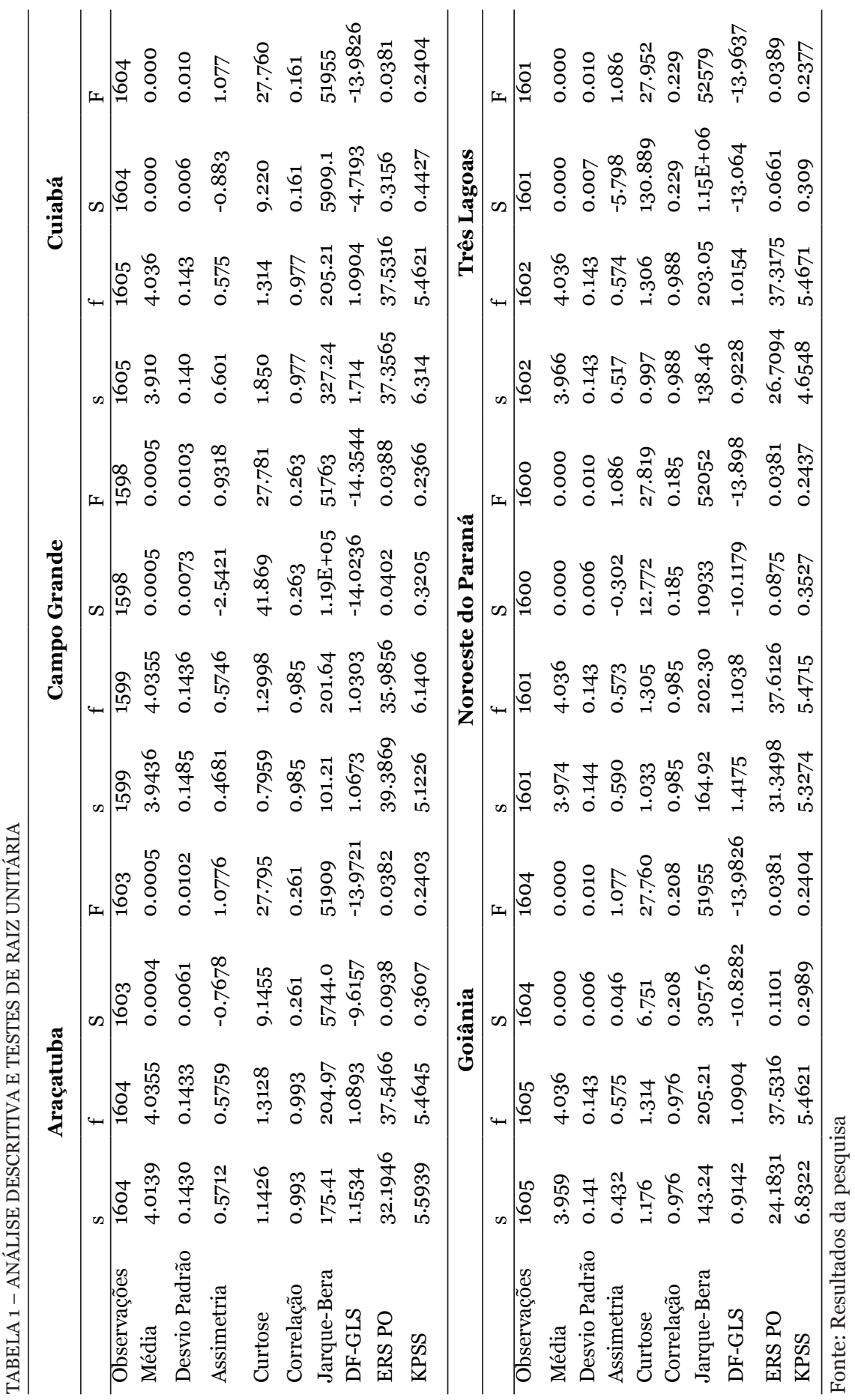


MONTEIRO, W. F.; RODRIGUES, M. A;.ALVES, A. F.; PARRÉ, J. L. A não efetividade do hedge...

Os testes DF-GLS, ponto ótimo de ERS e KPSS mostram que os preços à vista e futuros em log possuem raiz unitária, mas em primeira diferença são estacionários, nos níveis tradicionais de significância. Mesmo não reportadas na Tabela 1, todas as combinações entre constante e tendência foram realizadas, sendo que os resultados permanecem para a raiz unitária em nível e estacionariedade na primeira diferença. Portanto, são integradas de primeira ordem, precondição para testar a relação de cointegração, que requer mesma ordem de integração entre as séries.

TABELA 2 - TESTES DE COINTEGRAÇÃO DE JOHANSEN E JUSELIUS (1990)

\begin{tabular}{|c|c|c|c|c|c|c|}
\hline & \multirow{2}{*}{$\begin{array}{l}\text { VEC } \\
\text { Lags }\end{array}$} & $\lambda$ Traço & \multirow{2}{*}{$\begin{array}{c}\text { Estatística } \\
\lambda \text { Traço }\end{array}$} & \multicolumn{2}{|c|}{$\lambda$ Traço } & \multirow{2}{*}{$\begin{array}{l}\text { Vetor de Coin- } \\
\text { tegração } \\
\text { Normalizado }\end{array}$} \\
\hline & & $H_{0} \quad H_{1}$ & & $95 \%$ & $99 \%$ & \\
\hline \multirow[t]{2}{*}{ Araçatuba } & \multirow[t]{2}{*}{2} & $r=r=1$ & 271,54 & 19,96 & 24,60 & \multirow[t]{2}{*}[1,000,101-1,020]{} \\
\hline & & $r \leq r=2$ & 3,29 & 9,24 & 12,97 & \\
\hline \multirow{2}{*}{$\begin{array}{l}\text { Campo } \\
\text { Grande }\end{array}$} & \multirow[t]{2}{*}{2} & $r=r=1$ & 73,57 & 19,96 & 24,60 & \multirow[t]{2}{*}[1,000,339-1,061]{} \\
\hline & & $r \leq r=2$ & 3,06 & 9,24 & 12,97 & \\
\hline \multirow[t]{2}{*}{ Cuiabá } & \multirow[t]{2}{*}{2} & $r=r=1$ & 75,34 & 19,96 & 24,60 & \multirow[t]{2}{*}[1,000,399-1,069]{} \\
\hline & & $r \leq r=2$ & 5,88 & 9,24 & 12,97 & \\
\hline \multirow[t]{2}{*}{ Goiânia } & \multirow[t]{2}{*}{2} & $r=r=1$ & 103,4 & 19,96 & 24,60 & \multirow[t]{2}{*}[1,000,261-1,047]{} \\
\hline & & $r \leq r=2$ & 2,69 & 9,24 & 12,97 & \\
\hline \multirow[t]{2}{*}{$\begin{array}{l}\text { Noroeste } \\
\text { do Paraná }\end{array}$} & \multirow[t]{2}{*}{2} & $r=r=1$ & 128,07 & 19,96 & 24,60 & \multirow[t]{2}{*}[1,000,228-1,042]{} \\
\hline & & $r \leq r=2$ & 3,60 & 9,24 & 12,97 & \\
\hline \multirow[t]{2}{*}{$\begin{array}{l}\text { Três La- } \\
\text { goas }\end{array}$} & \multirow[t]{2}{*}{2} & $r=r=1$ & 92,53 & 19,96 & 24,60 & \multirow[t]{2}{*}[1,000,167-1,024]{} \\
\hline & & $r \leq r=2$ & 3,79 & 9,24 & 12,97 & \\
\hline
\end{tabular}

Fonte: Resultados da pesquisa

O teste do traço na Tabela 2 indica que as séries são cointegradas, isto é, os mercados à vista e futuro possuem relação de longo prazo. Portanto, um termo de correção de erro deve ser adicionado na modelagem. Foi rejeitada a hipótese de posto igual a zero e aceita a existência de pelo menos uma combinação linear. Esta é dada pela base, pois os valores do vetor de cointegração foram próximos a um. Desta forma, a suposição convergente de Fama e French (1987) entre os mercados, dada pela base, $\left(s_{t-1}-\delta f_{t-1}\right)$ tal que $\delta=1$ suposta por Kroner e Sultan (1993) se mantem. Para evitar perda informacional de longo prazo foi parametrizado o termo de correção de erro como média condicional. 
MONTEIRO, W. F.; RODRIGUES, M. A;.ALVES, A. F.; PARRÉ, J. L. A não efetividade do hedge...

A justificativa para usar modelos constantes nas covariâncias está sujeita ao hedger que permanece com a mesma razão entre sua exposição no mercado à vista em relação ao mercado futuro, sem calibragem do portfólio, diante do acréscimo de conteúdo informacional no mercado. Portanto, não seriam essenciais correlações variantes no tempo, com a finalidade de estimar a razão de hedge. Os testes de e Engle e Sheppard (2001) expostos na Tabela 3 rejeitam a forte suposição de correlações constantes para todas as regiões analisadas. Portanto, o cômputo das razões de hedge de forma dinâmica é primordial. Com base nesses resultados, as razões de hedge estimadas como constantes no tempo, isto é, estáticas, estão mal especificadas, pois as correlações no mercado de boi variam no tempo e devem ser modeladas. Portanto, foram estimadas por BEKK diagonal com alvo na variância.

TABELA 3 - TESTES DE ENGLE E SHEPPARD (2001) PARA CORRELAÇÃO CONSTANTE

\begin{tabular}{lcccccc}
\hline & Araçatuba & $\begin{array}{c}\text { Campo } \\
\text { Grande }\end{array}$ & Cuiabá & Goiânia & $\begin{array}{c}\text { Noroeste } \\
\text { do Paraná }\end{array}$ & $\begin{array}{c}\text { Três La- } \\
\text { goas }\end{array}$ \\
\hline E-S- Test (1) & $105.165^{* * * *}$ & $85.8307^{* * * *}$ & $81.4276^{* * *}$ & $93.8188^{* * * *}$ & $99.4866^{* * * *}$ & $69.6619^{* * * *}$ \\
E-S- Test (2) & $105.574^{* * *}$ & $86.5059^{* * * *}$ & $81.5036^{* * *}$ & $93.9363^{* * *}$ & $100.310^{* * *}$ & $69.6728^{* * *}$ \\
E-S- Test (3) & $105.590^{* * * *}$ & $87.0367^{* * *}$ & $82.5437^{* * *}$ & $93.9527^{* * *}$ & $100.329^{* * *}$ & $71.0696^{* * *}$ \\
E-S- Test (4) & $105.627^{* * *}$ & $87.5711^{* * *}$ & $83.0684^{* * *}$ & $94.0512^{* * *}$ & $100.775^{* * *}$ & $71.5119^{* * *}$ \\
E-S- Test (5) & $105.604^{* * *}$ & $98.0166^{* * * *}$ & $87.8300^{* * *}$ & $97.8408^{* * *}$ & $101.304^{* * *}$ & $72.7382^{* * *}$ \\
E-S- Test (6) & $105.631^{* * * *}$ & $98.5758^{* * * *}$ & $87.8098^{* * *}$ & $98.2019^{* * *}$ & $109.307^{* * *}$ & $73.3178^{* * *}$ \\
E-S- Test (7) & $111.659^{* * *}$ & $98.5520^{* * *}$ & $93.5713^{* * *}$ & $106.751^{* * *}$ & $110.812^{* * *}$ & $73.4790^{* * *}$ \\
E-S- Test (8) & $113.970^{* * *}$ & $100.643^{* * * *}$ & $93.5426^{* * *}$ & $107.227^{* * *}$ & $115.653^{* * *}$ & $195.231^{* * *}$ \\
E-S- Test (9) & $114.232^{* * *}$ & $101.055^{* * *}$ & $94.2539^{* * *}$ & $107.309^{* * *}$ & $115.783^{* * *}$ & $195.316^{* * *}$ \\
E-S- Test (10) & $114.260^{* * * *}$ & $101.878^{* * *}$ & $94.2419^{* * *}$ & $107.434^{* * *}$ & $117.039^{* * *}$ & $200.052^{* * *}$ \\
\hline
\end{tabular}

Fonte: Resultados da pesquisa

Algumas observações sobre os coeficientes na Tabela 4 BEKK merecem atenção. Todos foram estatisticamente significativos aos níveis tradicionais. Os valores com sinais contrários nas constantes $c_{1}$ e $c_{2}$ indicam o descompasso na média dos mercados. A velocidade do ajuste entre os preços e suas relações de longo prazo, mensurado pelo termo de correção de erro $b_{1}$ e $b_{2}$ são negativos na equação à vista, enquanto que positivo na equação do futuro. Implica que, em resposta a um desvio positivo, na relação de longo prazo, no período $t-1$, isto é, $S_{t-1}>F_{t-1}$, os preços à vista, no período subsequente, irão decrescer, enquanto que o futuro aumentará. 
MONTEIRO, W. F.; RODRIGUES, M. A;.ALVES, A. F.; PARRÉ, J. L. A não efetividade do hedge...

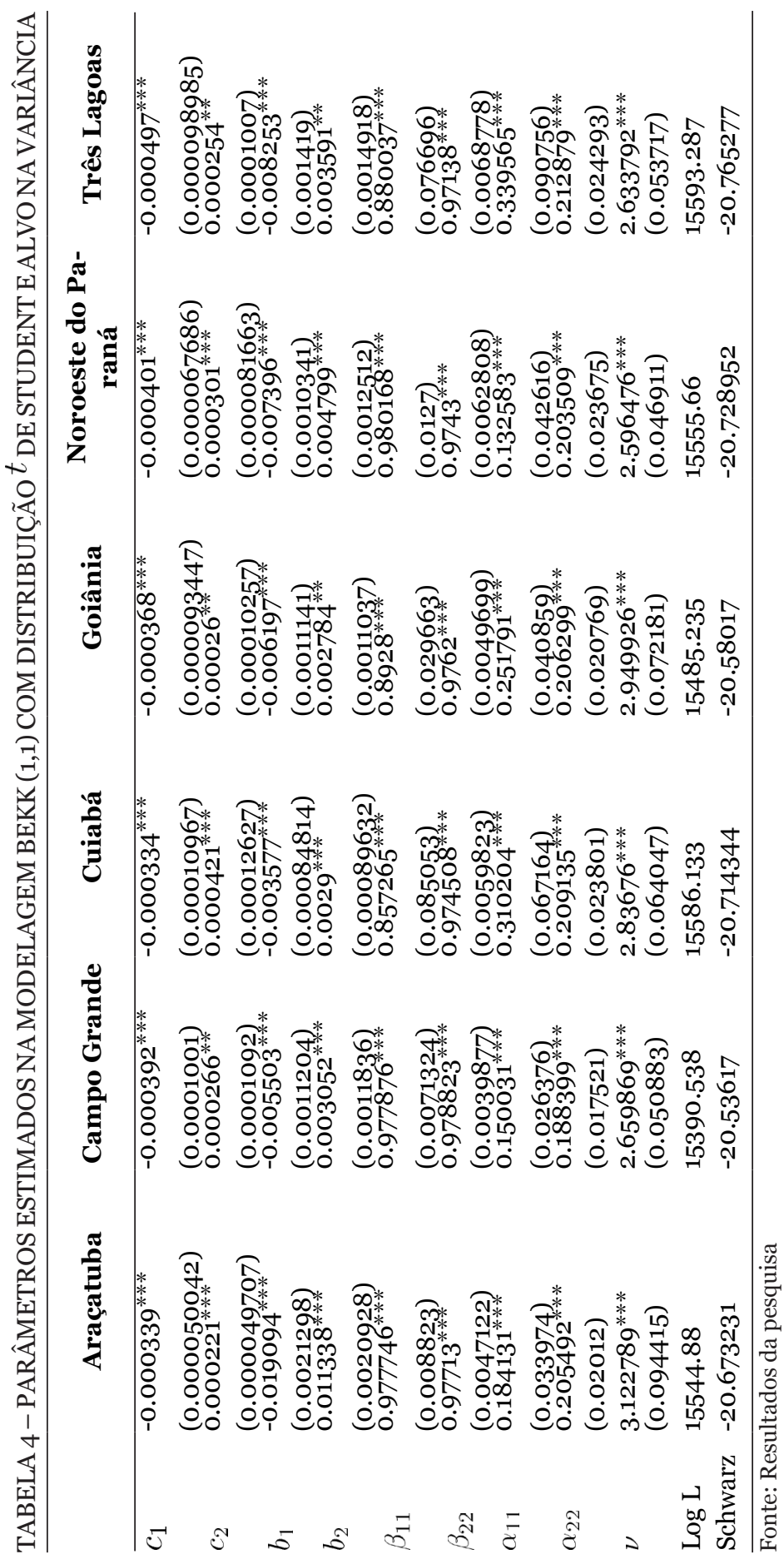


MONTEIRO, W. F.; RODRIGUES, M. A;.ALVES, A. F.; PARRÉ, J. L. A não efetividade do hedge...

A partir das estimativas descritas na Tabela 4, procedeu-se o cálculo das razões de hedge condicionais dentro e fora da amostra, expostas na Figura 1. É fácil notar sua mudança à medida que novas informações chegam ao mercado. $\mathrm{Na}$ maior parte do tempo, a proporção de contratos futuros tomados na redução do risco situou-se em valores inferiores a $50 \%$ da exposição no mercado físico, mas chegou a atingir valores negativos. Significa que para o hedger otimizar sua utilidade, seria necessário aumentar sua exposição no mercado à vista.

FIGURA 1 - RAZÕES DE HEDGE DENTRO E FORA DA AMOSTRA
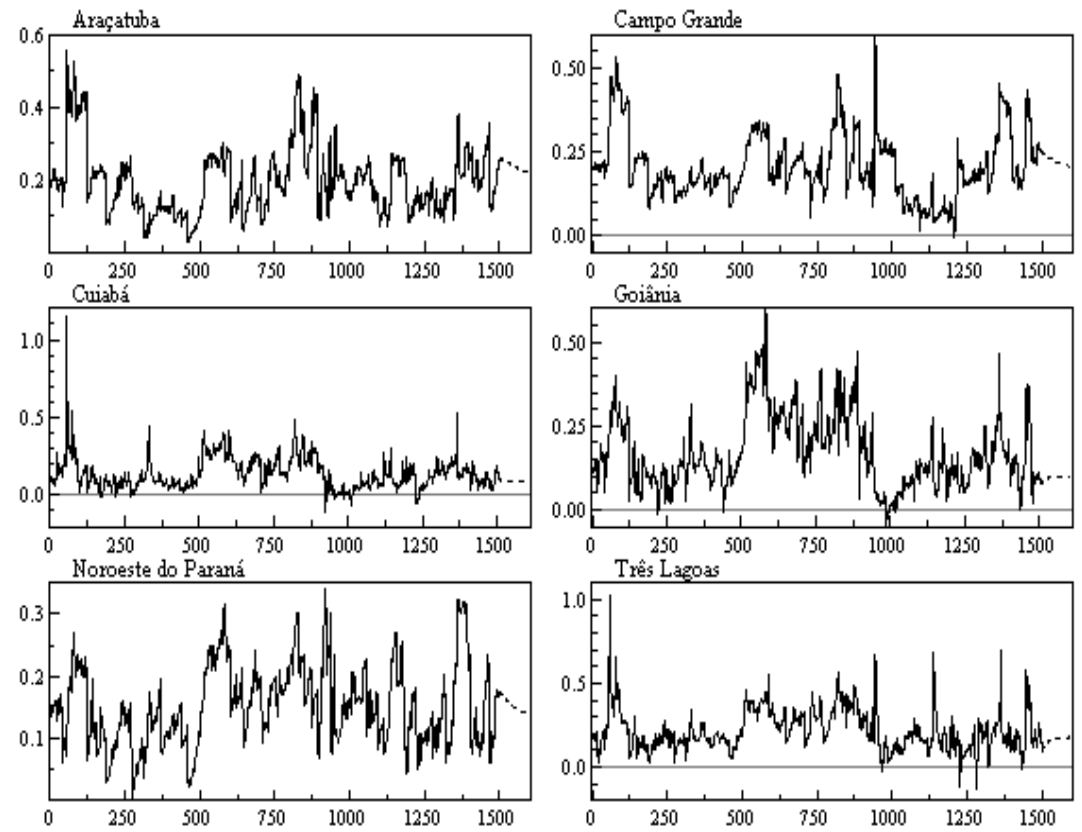

Fonte: Resultados da pesquisa

O usual na literatura é realizar comparações com outras estratégias que envolvem estimativas das razões de hedge de forma estática, por mínimos quadrados, processos autoregressivos, vetores de correção de erro. No entanto, não é seguido este procedimento, pois, a partir das evidências demonstradas anteriormente, não contemplariam as propriedades desejáveis nas estimativas como: estacionariedade das séries, obtidas com a primeira diferença, identificada por meio dos testes de raiz unitária; cointegração entre os retornos à vista e futuros, dada sua existência, então deve ser incorporado um vetor de correção de erro; bem como correlações variantes no tempo, uma vez que os testes de Engle e Sheppard (2001) indicaram que não são constantes. Desta forma, a comparação foi executada apenas com a opção de não fazer o hedge, pois a razão de hedge deve ser estimada por um método que as contemple, como a escolhida neste estudo. 
MONTEIRO, W. F.; RODRIGUES, M. A;.ALVES, A. F.; PARRÉ, J. L. A não efetividade do hedge...

Para os dados dentro da amostra, Tabela 5, em todas as praças, a redução de risco auferida foi positiva e próxima a zero, exceto Cuiabá que apresentou valor negativo mas também próximo de zero. Significa que a operação de hedge não foi efetiva em diminuir o risco dentro da amostra e chegou até mesmo aumentá-lo. Para os dados fora da amostra, as variâncias e covariâncias foram estimadas com um passo à frente. Os resultados foram semelhantes: aumento do risco, nas estimativas para Araçatuba e Noroeste do Paraná, e redução diminuta para as demais localidades. Deduz-se que considerar as propriedades desejáveis não foi suficiente para um hedge efetivo, portanto, o contrato futuro de boi gordo não cumpre com seu propósito de diminuição de risco aos hedgers. Apesar de não diminuir a variância, fica com utilidade aos especuladores deste mercado.

TABELA 5- EFETIVIDADE DO HEDGE

Araçatuba $\begin{gathered}\text { Campo } \\ \text { Grande }\end{gathered}$ Cuiabá $\quad$ Goiânia $\begin{gathered}\text { Noroeste } \\ \text { do Para- } \\ \text { ná }\end{gathered} \quad \begin{gathered}\text { Três } \\ \text { Lagoas }\end{gathered}$

Painel A - Dentro da Amostra

\begin{tabular}{lcccccc}
\hline $\begin{array}{l}\text { Variância } \\
\text { sem Hedge }\end{array}$ & $2.36 \mathrm{E}-06$ & $3.41 \mathrm{E}-06$ & $1.96 \mathrm{E}-06$ & $2.13 \mathrm{E}-06$ & $2.74 \mathrm{E}-06$ & $3.25 \mathrm{E}-06$ \\
$\begin{array}{l}\text { Variância } \\
\text { com Hedge }\end{array}$ & $2.27 \mathrm{E}-06$ & $3.27 \mathrm{E}-06$ & $1.97 \mathrm{E}-06$ & $2.09 \mathrm{E}-06$ & $2.70 \mathrm{E}-06$ & $3.22 \mathrm{E}-06$ \\
& & & & & & \\
Efetividade & 0.037156 & 0.038716 & -0.00474 & 0.017853 & 0.012891 & 0.011748
\end{tabular}

\section{Painel B - Fora da Amostra}

\begin{tabular}{lccccccc}
\hline $\begin{array}{l}\text { Variância } \\
\text { sem Hedge }\end{array}$ & $1.43 \mathrm{E}-06$ & $3.05 \mathrm{E}-06$ & $2.61 \mathrm{E}-06$ & $3.15 \mathrm{E}-06$ & $1.82 \mathrm{E}-06$ & $2.16 \mathrm{E}-06$ \\
$\begin{array}{l}\text { Variância } \\
\text { com Hedge }\end{array}$ & $1.46 \mathrm{E}-06$ & $2.92 \mathrm{E}-06$ & $2.53 \mathrm{E}-06$ & $3.09 \mathrm{E}-06$ & $1.91 \mathrm{E}-06$ & $2.13 \mathrm{E}-06$ \\
Efetividade & -0.02016 & 0.04093 & 0.029524 & 0.017973 & -0.05099 & 0.01733 \\
\hline
\end{tabular}

Fonte: Resultados da pesquisa 


\section{Considerações Finais}

As modelagens devem considerar os retornos das séries e não os preços em nível, uma vez que a estacionariedade é atingida na primeira diferença. Os retornos à vista e futuros possuem pelo menos uma combinação linear, assim, é necessária à adição de um termo de correção de erro para garantir a relação de longo prazo, pois são cointegrados. Mesmo que sejam considerados, é essencial que a razão de hedge mude com o tempo, uma vez que as correlações e variâncias não são constantes. As evidências enunciadas indicam má especificação nas modelagens das razões de hedge do boi gordo caso não sejam consideradas. Portanto, foram estimadas a partir do modelo BEKK diagonal com alvo na variância, adicionado do termo de correção de erro parametrizado como média condicional.

Os resultados indicaram o mercado futuro como menos estável que os à vista e, se comparados os desvios padrões, estes foram similares nas diversas praças e inferiores ao mercado futuro. Diferente da maioria das séries financeiras, ocorre assimetria negativa nos retornos à vista e positiva nos futuros, bem como excesso de curtose dos retornos futuros maiores que os à vista. Estas evidências justificaram o uso da distribuição $t$ de student. Em todas as séries a correlação dos retornos foi baixa, implicou razões de hedge inferiores a 50\%, mas chegaram a atingir valores negativos. Para os dados dentro da amostra, a redução do risco foi próxima a zero e com razões estimadas um passo à frente, houve aumento do risco ou redução diminuta. Deduz-se que o hedge não foi efetivo. Portanto, o contrato futuro de boi gordo não aufere diminuição de risco aos hedgers, em análise realizada com retornos. No entanto, continua útil para fins especulativos.

Como os resultados apresentaram não efetividade do hedge, é possível supor que este mercado não é eficiente. Uma das possíveis formas de testá-la, é verificar uma relação [ $\left[\begin{array}{lll}1 & 0 & -1\end{array}\right]$ para o vetor de cointegração, isto é, a base no período $t-1$. Se for falsa, assim como a base não estacionária, podem ser explicações plausíveis à baixa diminuição de risco proporcionado no mercado futuro de boi gordo. Mas fica como sugestão para pesquisa futura.

\section{Referências Bibliográficas}

Abitante, K. G. (2008) "Co-integração entre os mercados spot e futuro: evidências dos mercados de boi gordo e soja." Revista de Economia e Sociologia Rural, v. 46, n. 1, p. 75-96.

Alexander, C. (2008) "Market Risk Analysis: Pricing, Hedging and Trading Financial Instruments.” 1. ed. West Sussex: John Wiley \& Sons.

Bitencourt, W. A.; Silva, W. S.; Sáfadi, T. (2006) "Hedge dinâmicos: uma evidência para os contratos futuros brasileiros." Organizações Rurais \& Agroindustriais, v. 8, n. 1, p. $71-78$. 
MONTEIRO, W. F.; RODRIGUES, M. A;.ALVES, A. F.; PARRÉ, J. L. A não efetividade do hedge...

BM\&F. (2007) "Especificações do contrato futuro de boi gordo denominado em reais." São Paulo.

Brooks, C. (2008) "Introductory Econometrics for Finance.” 2. ed. Cambrige: Cambridge University Press.

Ederington, L. H. (1979) "The hedging performance of the new futures markets." The Journal of Finance, $v .34, n .1, p .157-170$.

Engle, R. F.; Kroner, K. F. (1995) “Multivariate simultaneous generalized ARCH.” Econometric Theory, v. 11, n. 1, p. 122-150.

Engle, R. F.; Mezrich, J. (1996) Garch for Groups. Risk, v. 9, n. 8, p. 36-4O.

Engle, R. F.; Sheppard, K. (2001) “Theoretical and Empirical properties of Dynamic Conditional Correlation Multivariate GARCH.” [S.l.]

Fama, E. F.; French, K. R. (1987) “Commodity futures prices: Some evidence on forecast power, premiums, and the theory of storage." Journal of Business, $v$. 60, n. 1, p. 55-73.

Fileni, D. H.; Marques, P. V.; Machado, H. M. (1999) "O risco de base e a efetividade do hedge para o agronegócio do café em minas gerais.” Organizações Rurais \& Agroindustriais, v. 1, n. 1, p. 42-50.

Ford, J. L.; Pok, W. C.; Poshakwale, S. (2006) "Dynamic vs. Static Stock Index Futures Hedging: A Case Study for Malaysia.” [S.l.]

Gaio, L. E.; Castro, L. G. ; Oliveira, A. R. de. (2005) “Causalidade e elasticidade na transmissão de preço do boi gordo entre regiões do Brasil e a bolsa de mercadorias \& futuros BM\&F." Organizações Rurais \& Agroindustriais, v. 7, n. 3, p. 282-297.

Gonçalves, D. F. et al. (2008) “Co-integração, causalidade e efetividade do hedge para preços do contrato de boi gordo para o noroeste do Paraná.” Informe Gepec, v. 12, n. 1, p. 1-14.

Guimarães, R. L. (2005) "Razão ótima de hedge em função do horizonte de hedge e da periodicidade dos dados: uma aplicação no mercado de boi gordo brasileiro." 88 p. Dissertação (Mestrado em Finanças e Economia Empresarial) - Escola de Economia de São Paulo, Fundação Getúlio Vargas, São Paulo.

Herbst, A. F.; Kare, D. D.; Caples, S. C. (1989) "Hedging effectiveness and minimum risk hedge ratios in the presence of autocorrelation: Foreign currency futures." Journal of Futures Markets, v. 9, n. 3, p. 185-197.

Hull, J. (2006) "Options, Futures, and Other Derivatives." 6. ed. New Jersey: Prentice Hall.

IBGE. (2008) “Pesquisa pecuária municipal.” INSTITUTO BRASILEIRO DE GEOGRAFIA E ESTATÍSTICA.

Johansen, S.; Juselius, K. (1990) "Maximum likelihood estimation and inference on cointegration-with applications to the demand for money." Oxford Bulletin of Economics and Statistics, v. 52, n. 2, p. 169-210.

Johnson, L. L. (1960) "The theory of hedging and speculation in commodity futures." The Review of Economic Studies, v. 27, n. 3, p. 139-151. 
MONTEIRO, W. F.; RODRIGUES, M. A;.ALVES, A. F.; PARRÉ, J. L. A não efetividade do hedge...

Kroner, K. F.; Sultan, J. (1993) "Time-varying distributions and dynamic hedging with foreign currency futures." The Journal of Financial and Quantitative Analysis, v. 28, n. 4, p. 535-551.

Lien, D.; Tse, Y. K. (2002) “Some recent developments in futures hedging.” Journal of Economic Surveys, v. 16, n. 3, p. 357-96.

Lien, D.; Zhang, M. (2008) “A survey of emerging derivatives markets.” Emerging Markets Finance and Trade, v. 44, n. 2, p. 39-69.

Martins, A. G.; Aguiar, D. R. D. (2004) "Efetividade do hedge de soja em grão com contratos futuros de diferentes vencimentos na Chicago Board of Trade." Revista de Economia e Agronegócio, v. 2, n. 4, p. 449-47.

Müller, C. A. S.; Moura, A. D.; Lima, J. E. (2008) “Análise comparativa entre estratégias de hedge com base em modelos estáticos e dinâmicos para contratos futuros de café arábica." In: CONGRESSO BRASILEIRO DE ECONOMIA E SOCIOLOGIA RURAL, XLVI., 2008, Rio Branco. Anais... Rio Branco: Sociedade Brasileira de Economia, Administração e Sociologia Rural, p. 21.

Myers, R.; Thompson, S. (1989) “Generalized optimal hedge ratio estimation.” American Journal of Agricultural Economics, v. 71, n. 4, p. 858-868.

Nogueira, F. T. P.; Aguiar, D. R. D.; Lima, J. E. (2002) "Efetividade do hedge no mercado brasileiro de café arábica.” Resenha BM\&F, n. 15O, p. 11.

Yang, W.; Allen, D. E. (2005) "Multivariate GARCH hedge ratios and hedging effectiveness in australian futures markets." Accounting and Finance, v. 45, $n$. 2, p. 301-21.

Ye, T.; Chen, Z. (2006) “The Hedging Effectiveness of Currency Futures.” 4o p. Dissertação (Mestrado em Artes) - Faculty of Business Administration, Simon Fraser University, Burnaby.

Zilli, J. B. et al. (2008) “Análise da cointegração e causalidade dos preços de boi gordo em diferentes praças nas regiões sudeste e centro-oeste do brasil.” Revista de Economia Agrícola, v. 55, n. 2, p. 105-119.

Recebido em: 24 de setembro de 2012 Aceito em: 31 de março de 2013 